\title{
RGD-modified poly(D,L-lactic acid) nanoparticles enhance tumor targeting of oridonin
}

\author{
This article was published in the following Dove Press journal: \\ International Journal of Nanomedicine \\ 6 January 2012 \\ Number of times this article has been viewed
}

Jie Xu
Ji-Hui Zhao
Ying Liu
Nian-Ping Feng
Yong-Tai Zhang

School of Pharmacy, Shanghai University of Traditional Chinese Medicine, Shanghai, People's Republic of China
Correspondence: Nian-Ping Feng Department of Pharmaceutics, School of Pharmacy, Shanghai University of Traditional Chinese Medicine, I200 Cailun Road, Zhangjiang Hi-Tech Park, Pudong New District, Shanghai 20I 203, People's Republic of China

$\mathrm{Tel}+862151322198$

Fax +862151322198

Email npfeng@hotmail.com
Objective: The purpose of this study was to develop an active targeting strategy to improve the therapeutic antitumor efficacy of oridonin (ORI), the main active ingredient in the medicinal herb Rabdosia rubescens.

Methods: A modified spontaneous emulsification solvent diffusion method was used to prepare the ORI-loaded atactic poly(D,L-lactic acid) nanoparticles (ORI-PLA-NPs). Surface crosslinking with the peptide Arg-Gly-Asp (RGD) further modified the ORI-PLA-NPs, generating ORI-PLA-RGD-NPs. The NPs were characterized and release experiments were performed in vitro. The pharmacokinetics, tissue distribution, and antitumor activity of the NPs were studied in mice bearing hepatocarcinoma 22 (H22)-derived tumors.

Results: The ORI-PLA-NPs and ORI-PLA-RGD-NPs were smooth, sphere-like, and relatively uniform in size. The RGD surface modification slightly increased the mean particle size $(95.8 \mathrm{~nm}$ for ORI-PLA-NPs versus $105.2 \mathrm{~nm}$ for ORI-PLA-RGD-NPs) and considerably altered the surface electrical property $(-10.19 \mathrm{mV}$ for ORI-PLA-NPs versus $-21.95 \mathrm{mV}$ for ORI-PLA-RGD-NPs), but it had no obvious influence on ORI loading $(8.23 \% \pm 0.35 \%$ for ORI-PLA-NPs versus $8.02 \% \pm 0.38 \%$ for ORI-PLA-RGD-NPs), entrapment efficiency $(28.86 \% \pm 0.93 \%$ for ORIPLA-NPs versus $28.24 \% \pm 0.81 \%$ for ORI-PLA-RGD-NPs), or the release of ORI. The pharmacokinetic properties of free ORI were improved by encapsulation in NPs, as shown by increased area under the concentration-time curve $\left(11.89 \pm 0.35 \mu \mathrm{g} \cdot \mathrm{mL}^{-1} \cdot \mathrm{h}\right.$ for ORI solution versus $22.03 \pm 0.01 \mu \mathrm{g} \cdot \mathrm{mL}^{-1} \cdot \mathrm{h}$ for ORI-PLA-RGD-NPs) and prolonged mean retention time $(2.03 \pm 0.09$ hours for ORI solution versus $8.68 \pm 0.66$ hours for ORI-PLA-RGD-NPs). In the tissue distribution study, more ORI targeted tumor tissue in the mice treated with ORI-PLA-RGD-NPs than with ORI-PLA-NPs or ORI solution. Consistent with these observations, ORI-PLA-RGDNPs showed greater antitumor efficacy than ORI-PLA-RGD-NPs or ORI solution, as reflected by the decreased tumor growth and the prolonged survival time of mice bearing $\mathrm{H} 22$ tumors.

Conclusion: The tumor-targeting efficiency and subsequent antitumor efficacy of ORI is increased by incorporation into ORI-PLA-RGD-NPs.

Keywords: ORI, antitumor activity, RGD, poly(D,L-lactic acid), nanoparticles

\section{Introduction}

The medicinal herb dong ling cao (Rabdosia rubescens) is native to the Yellow River valley in China and is used as a folk remedy for tonsillitis and a variety of cancers. The main active ingredient isolated from $R$. rubescens is oridonin (ORI), a monomeric ent-kaurane diterpenoid compound. Over the past 30 years, ORI has been successfully used for the treatment of liver cancer and esophageal carcinoma. ${ }^{1}$ In addition, pharmacological experiments have shown ORI to have broad-spectrum antitumor activity, suggesting that ORI could play a more prominent role in cancer 
therapy. Investigations into the mechanisms underlying its antitumor activity have reported that ORI plays a role in the growth and survival of a variety of cancer cell lines, including cell cycle arrest of K1735M2, DU-145, MCF-7, and MCF-10A cells; $;^{2-4}$ apoptosis of U937 and A375-S2 cells; ;,6 and autophagy of A431 cells. ${ }^{7}$ In addition, ORI has synergistic effects on cell cycle arrest and apoptosis of HEP-2, L929, and SMMC-7721 cells; ${ }^{8-10}$ and on cell apoptosis and autophagy in the HeLa cells. ${ }^{11,12}$ However, the clinical application of ORI is considerably limited by its poor water solubility and unfavorable pharmacokinetic properties such as low oral bioavailability, which result in the need for high doses and thus increase the potential for side effects. ${ }^{13}$ Although the oral bioavailability of ORI was shown to be increased 2.2-fold by a self-microemulsifying delivery system compared with ORI in suspension, ${ }^{14,15}$ intravenous targeted delivery systems show greater potential for improving antitumor therapeutic efficacy while simultaneously reducing side effects. The antitumor efficacy of ORI was reported to be improved greatly by ORI nanosuspensions and by the use of passive targeting nanoscale delivery systems such as solid lipid nanoparticles (NPs), poly(D,L-lactic acid) (PLA) NPs, and poly(epsilon-caprolactone)-poly(ethylene oxide)poly(epsilon-caprolactone) copolymer NPs. ${ }^{16-19}$ PLA, a biodegradable polymer approved by the US Food and Drug Administration for biomedical applications, has been studied as a particulate drug carrier since 1970. PLA-NPs copolymerized with polyethylene glycol and surface modified with ligands such as Arg-Gly-Asp (RGD) peptides show increased drug loading, reduced burst effect, prolonged in vivo residence time, decreased recognition and engulfment by macrophages, and improved targeting of tumor cells. ${ }^{20-23}$ More recently, PLA polymer stars have been synthesized to improve hydrophilicity and control degradation of PLA. Using PLA polymer star micelles as carriers, the rate of release of the model drug chlorambucil could be effectively controlled by altering the $\mathrm{pH}$ of the environment. ${ }^{24,25}$ In the present study, the authors sought to further improve the therapeutic antitumor efficacy of ORI by constructing an active targeting nanoscale delivery system. For this, an RGD motif was incorporated onto the surface of PLA-NPs to improve the tissue targeting efficiency of ORI.

\section{Materials and methods \\ Materials}

ORI raw material (Figure 1) was obtained from Hangzhou Huadong Medicine Group Kangrun Pharmaceutical Co, Ltd (Anji, Zhejiang, China). Atactic PLA, with an average

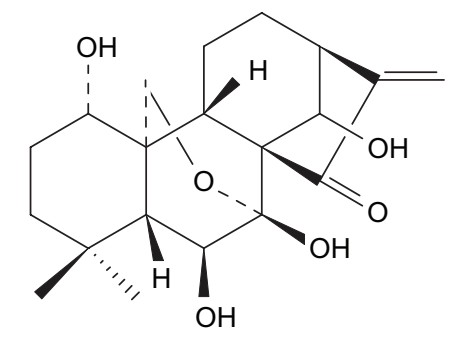

Figure I Chemical structure of oridonin.

molecular weight of 15,000 Da and polydispersity index of 1.5, was purchased from Shandong Institute of Medical Instruments (Jinan, Shandong, China). Poloxamer 188 was provided by BASF (Pluronic ${ }^{\circledR}$ F68; Ludwigshafen, Germany). Arg-Gly-Asp-L-Phe-Lys (RGDfk), N-(3dimethylaminopropyl)- $N$ '-ethylcarbodiimide hydrochloride (EDC-HCl), and $N$-hydroxysulfosuccinimide (sulfo-NHS) were purchased from GL Biochem (Shanghai) Ltd (Shanghai, China). Other chemicals were of high-performance liquid chromatography (HPLC) or analytical grade.

The Experimental Animal Center of Shanghai University of Traditional Chinese Medicine, Shanghai, China, supplied the male Kunming mice (mean body weight $20 \pm 2 \mathrm{~g}$ ). All care and handling of the animals was performed with the approval of the Institutional Authority for Laboratory Animal Care of Shanghai University of Traditional Chinese Medicine. The Shanghai Laboratory Animal Center, Academia Sinica, Shanghai, China, provided the hepatocarcinoma 22 (H22) cell line (murine sarcoma).

\section{Preparation and characterization of ORI-PLA-RGD-NPs}

The ORI-PLA-NPs were prepared by a spontaneous emulsification solvent diffusion method, slightly modified from that previously described. ${ }^{26-29} \mathrm{In}$ brief, $12 \mathrm{mg}$ of ORI and $50 \mathrm{mg}$ of PLA were dissolved in $20 \mathrm{~mL}$ of solvent (acetone:ethanol $3: 2, \mathrm{v} / \mathrm{v}$ ) for 5 minutes with magnetic stirring at $50 \mathrm{rpm}$. The resulting oil phase was mixed thoroughly with $30 \mathrm{~mL}$ of aqueous Pluronic F68 (1\% v/v) by magnetic stirring at $50 \mathrm{rpm}$ for 5 minutes. The organic solvents were removed and the volume further reduced to $5 \mathrm{~mL}$ at $40^{\circ} \mathrm{C}$ under reduced pressure. The sample was filtered through a $0.2 \mu \mathrm{m}$ pore size Millipore ${ }^{\circledR}$ filter (Millipore, Billerica, MA) to yield the final suspension of ORI-PLA-NPs.

Surface peptide cross-linking was used to modify ORIPLA-NPs. To $10 \mathrm{~mL}$ of the ORI-PLA-NP solution was separately added $15 \mathrm{mg}$ of EDC-HCl and $15 \mathrm{mg}$ of sulfo-NHS. The $\mathrm{pH}$ was adjusted to 6.0 with $0.1 \mathrm{M} 2$-( $N$-morpholino) 
ethanesulfonic acid ([MES] J\&K Chemical (China) Ltd, Shanghai, China) and the system was kept at room temperature for 12 hours with gentle stirring. The active intermediate was isolated with a Sephadex ${ }^{\circledR}$ G-50 minicolumn (Pharmacia Biotech, Uppsala, Sweden). Two milligrams of RGDfk was added to the solution of active intermediate and the solution was kept on ice for 24 hours with gentle stirring. The sample was purified with a Sephadex G-50 minicolumn to yield the final suspension of ORI-PLA-RGD-NPs.

A transmission electron microscope, a scanning electron microscope, and a Zetasizer (Nano ZS90; Malvern Instruments Ltd, Worcestershire, UK) were used to analyze the morphology, particle size, and zeta potential, respectively, of the prepared ORI-PLA-NPs and ORI-PLA-RGD-NPs.

The efficiency of ORI loading and entrapment in ORIPLA-NPs and ORI-PLA-RGD-NPs was calculated using the following equations:

ORI loading $=\frac{\text { weight of ORI in NPs }}{\text { weight of NPs }} \times 100 \%$

ORI entrapment efficiency $=\frac{\text { weight of ORI in NPs }}{\text { weight of ORI added }} \times 100 \%$

Quantities of free or entrapped ORI were determined by HPLC. For the NP suspensions, dimethyl sulfoxide was added to dissolve the NPs, and the suspensions were ultracentrifuged for 2 hours at $4^{\circ} \mathrm{C}$. The supernatants were taken for HPLC.

\section{ORI release experiments}

Experiments were conducted to determine the release of ORI from ORI-PLA-NPs and ORI-PLA-RGD-NPs over time. The NP suspension $(10 \mathrm{~mL})$ was placed in a dialysis bag (molecular weight cutoff 10,000 Da) and dialyzed against $190 \mathrm{~mL}$ of normal saline at $37^{\circ} \mathrm{C}$ with gentle shaking. At predetermined time points, a $5 \mathrm{~mL}$ sample was removed and replaced with $5 \mathrm{~mL}$ of normal saline equilibrated to $37^{\circ} \mathrm{C} \pm 0.5^{\circ} \mathrm{C}$. The ORI content of the withdrawn samples was determined by HPLC.

\section{Pharmacokinetic and biodistribution studies}

The H22 tumor model in male Kunming mice was established as reported previously. ${ }^{10}$ In brief, the mice were inoculated with H22 cells subcutaneously in the axillary region. The mice were maintained under standard laboratory conditions until the diameters of the tumors reached $0.6-0.8 \mathrm{~cm}$. For pharmacokinetic and tissue distribution studies, 144 H22 tumor-bearing mice were divided randomly into three groups of 48 mice and were designated to receive ORI in solution, ORI-PLA-NPs, or ORI-PLA-RGD-NPs as a single intravenous injection (final ORI content $20 \mathrm{mg} \mathrm{kg}^{-1}$ ). Blood was withdrawn at $0.167,0.5,1,2,3,4,6,8,12,18,24$, and 36 hours after injection, and four mice per group were sacrificed at each sampling time point. The heart, liver, spleen, lungs, kidneys, and tumor were removed and homogenized. The ORI content in plasma samples and tissue homogenates was determined by HPLC.

\section{In vivo antitumor studies}

To evaluate the in vivo antitumor effect of ORI in solution or loaded in the NPs, four groups of six H22 tumor-bearing mice received intravenous injections of normal saline, ORI solution, ORI-PLA-NPs, or ORI-PLA-RGD-NPs (final ORI content $20 \mathrm{mg} \mathrm{kg}^{-1}$ ) once daily for 10 days. Mice were sacrificed 24 hours after the last injection and the tumors were removed and weighed. The tumor volumes were calculated using the following equation:

$$
\text { Tumor volume }=\frac{a b^{2}}{2}
$$

where $\mathrm{a}$ and $\mathrm{b}$ represent the long and short axes of the tumor, respectively.

To evaluate the effect of the NPs on organ pathology, organs from both the treated and the control mice were prepared, stained with hematoxylin and eosin, and subjected to histopathological analysis.

A survival study was conducted with four groups of twelve $\mathrm{H} 22$ tumor-bearing mice randomized to receive the test articles exactly as described for the antitumor experiments. Normal saline, ORI solution, ORI-PLA-NPs, or ORI-PLA-RGD-NPs were administered once daily intravenously. Survival was monitored daily.

\section{Data analysis}

Noncompartmental pharmacokinetic analysis of plasma ORI concentrations versus time was performed with Bioavailability Program Package software (BAPP v 2.0; Center for Metabolism and Pharmacokinetics, China Pharmaceutical University, Nanjing, China). One-way analysis of variance and Fisher's least significant difference $t$-tests were used for the statistical analysis of data obtained in the in vivo antitumor experiments, and the log-rank test was used for multiple comparisons of the median survival times. 


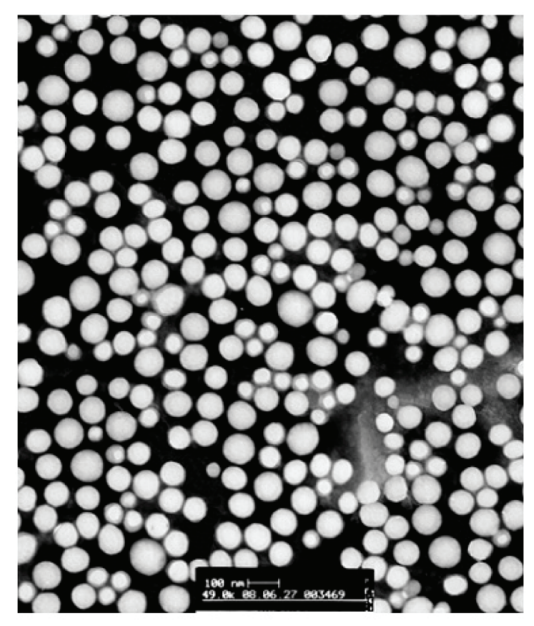

ORI-PLA-NPs

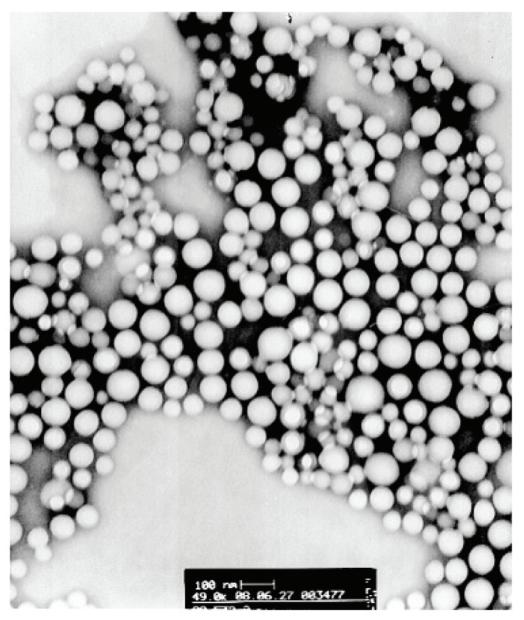

ORI-PLA-RGD-NPS

Figure 2 Transmission electron microscopy images of oridonin-loaded atactic poly(D,L-lactic acid) nanoparticles (ORI-PLA-NPs) and ORI-PLA-NPs further modified by surface cross-linking with the peptide Arg-Gly-Asp (ORI-PLA-RGD-NPs) (magnification 43,000x).

\section{Results and discussion}

\section{Characterization of ORI-PLA-RGD-NPs}

As shown by the transmission electron microscopy and scanning electron microscopy images in Figures 2 and 3 , the prepared NPs were smooth, sphere-like, and relatively uniform in size. The mean particle sizes, polydispersity indices, and zeta potentials were $95.8 \pm 1.2 \mathrm{~nm}$, $0.075 \pm 0.008$, and $-10.19 \pm 0.62 \mathrm{mV}$, respectively, for ORI-PLA-NPs and $105.2 \pm 0.7 \mathrm{~nm}, 0.142 \pm 0.011$, and $-21.95 \pm 1.04 \mathrm{mV}$, respectively, for ORI-PLA-RGD-NPs. The surface modification of ORI-PLA-NPs with RGD is likely responsible for the slight increase in the particle size and polydispersity index and for the considerable change in zeta potential. In contrast, the RGD modification had no obvious influence on ORI loading $(8.23 \% \pm 0.35 \%$ for ORI-PLA-NPs versus $8.02 \% \pm 0.38 \%$ for ORI-PLARGD-NPs) or entrapment efficiency $(28.86 \% \pm 0.93 \%$ for ORI-PLA-NPs versus $28.24 \% \pm 0.81 \%$ for ORI-PLARGD-NPs).

\section{ORI release experiments}

The ORI release profiles of ORI-PLA-NPs and ORI-PLARGD-NPs are shown in Figure 4. The profiles are virtually superimposable, and the release data of both ORI-PLA-NPs and ORI-PLA-RGD-NPs fit the Weibull distribution well ( $r=0.9971$ for ORI-PLA-NPs versus $r=0.9952$ for ORIPLA-RGD-NPs). Two-stage release behavior was observed in both profiles, with the burst release of ORI (25\%) occurring in the first 4 hours, followed by the sustained release from 5 to 72 hours (50\%). This behavior might be attributed to the initial rapid release of ORI from the surface and external layer of the NPs, followed by sustained release from the internal layer through diffusion combined with erosion of the NPs. ${ }^{30}$ In a previous study, the release profile of ORI-PLA-NPs was

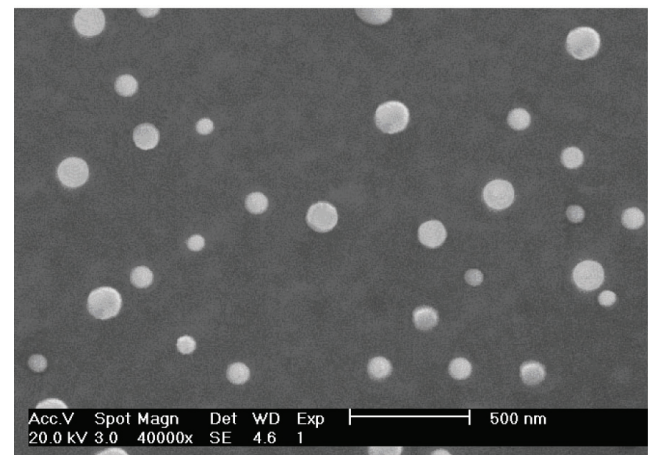

ORI-PLA-NPs

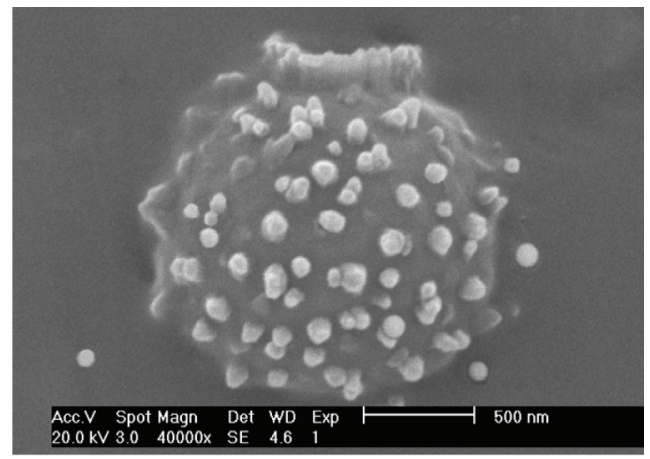

ORI-PLA-RGD-NPs

Figure 3 Scanning electron microscopy images of oridonin-loaded atactic poly(D,L-lactic acid) nanoparticles (ORI-PLA-NPs) and ORI-PLA-NPs further modified by surface cross-linking with the peptide Arg-Gly-Asp (ORI-PLA-RGD-NPs) (magnification 40,000x). 


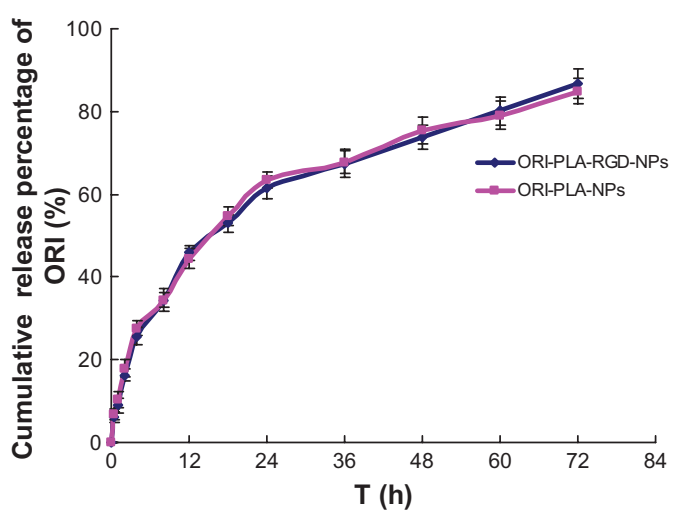

Figure 4 Release of oridonin (ORI) over time from ORI-loaded atactic poly(D,Llactic acid) nanoparticles (ORI-PLA-NPs) and from ORI-PLA-NPs further modified by surface cross-linking with the peptide Arg-Gly-Asp (ORI-PLA-RGD-NPs).

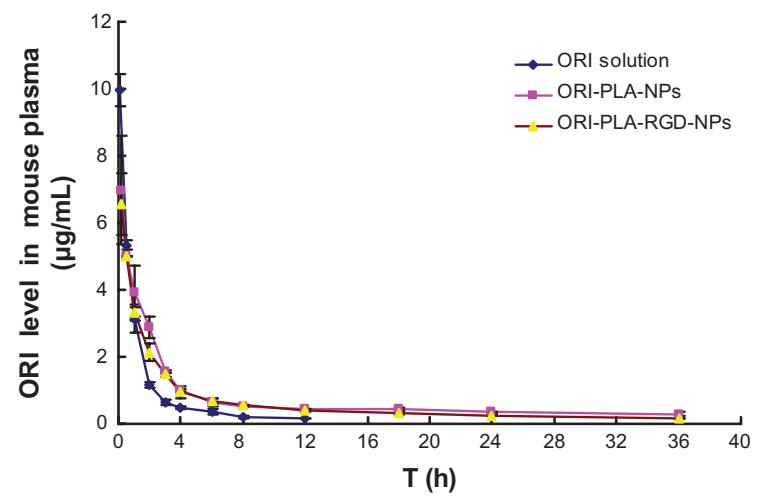

Figure 5 Mean oridonin (ORI) concentrations in mouse plasma versus time after a single intravenous injection of ORI solution, ORI-loaded atactic poly(D,L-lactic acid) nanoparticles (ORI-PLA-NPs), or ORI-PLA-NPs further modified by surface crosslinking with the peptide Arg-Gly-Asp (ORI-PLA-RGD-NPs).
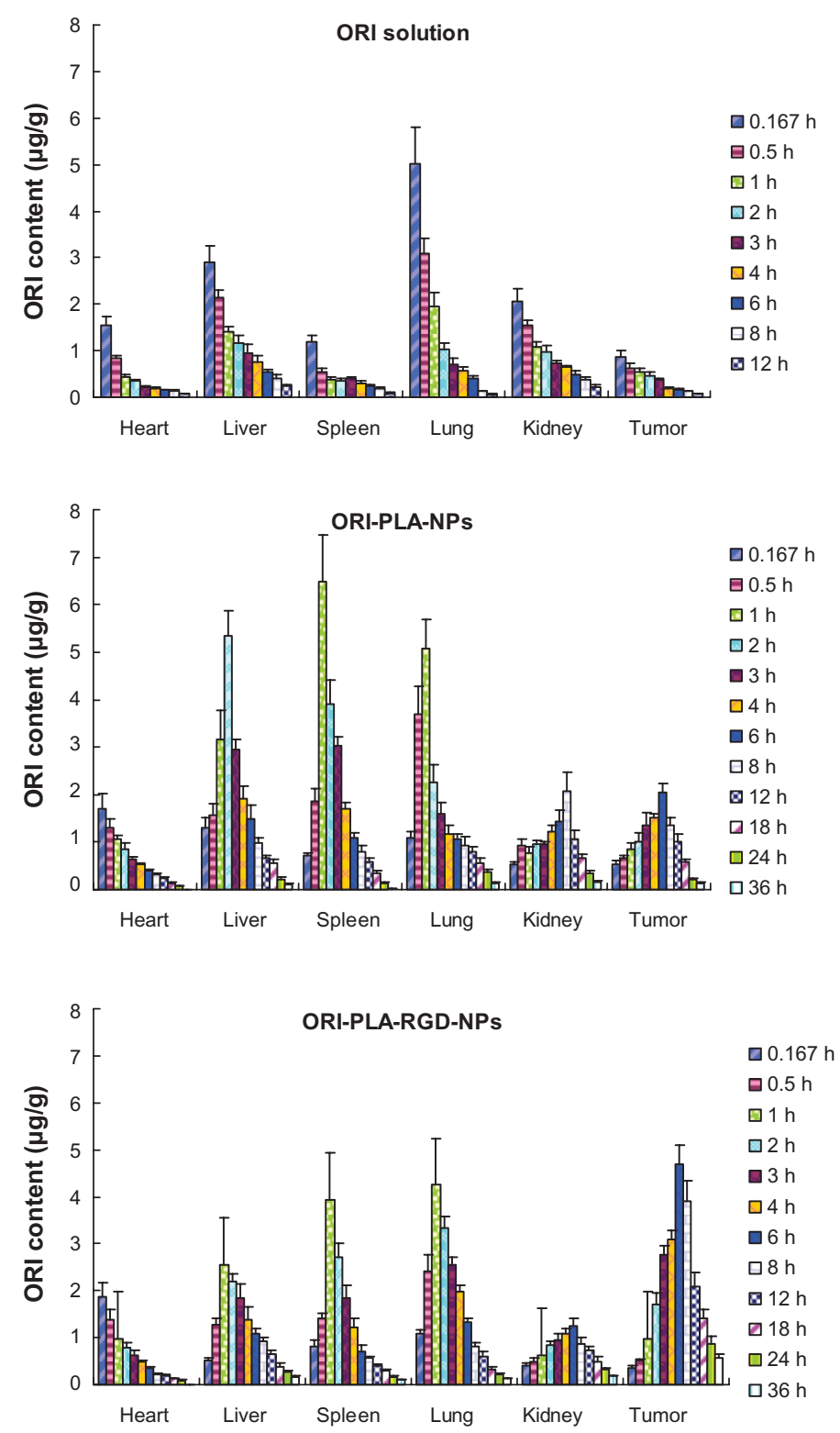

Figure 6 Mean concentrations of oridonin (ORI) in tissues of tumor-bearing mice after a single injection of ORI solution, ORI-loaded atactic poly(D,L-lactic acid) nanoparticles (ORI-PLA-NPs), or ORI-PLA-NPs further modified by surface cross-linking with the peptide Arg-Gly-Asp (ORI-PLA-RGD-NPs).

Abbreviation: h, hours. 
reported to fit the Higuchi model well, but the burst effect was also observed and the release mechanism was the same as that suggested earlier. ${ }^{18}$

\section{Pharmacokinetics and tissue distribution}

To assess the in vivo characteristics of the NPs, ORI solution, ORI-PLA-NPs, and ORI-PLA-RGD-NPs were administered to mice as single intravenous injections of a dose equivalent to $20 \mathrm{mg} \mathrm{kg}^{-1}$ of ORI. The corresponding mean plasma concentrations versus time curves and the tissue distribution profiles are shown in Figures 5 and 6, respectively. The individual pharmacokinetic parameters are given in Table 1.

As indicated by the plasma concentration versus time profiles, the NPs prolonged the retention time and increased the area under the concentration-time curve (AUC) compared with ORI solution. This result was confirmed by comparing the pharmacokinetic parameters. Increases were observed in the AUC $\left(22.03 \pm 0.01 \mu \mathrm{g} \cdot \mathrm{mL}^{-1} \cdot \mathrm{h}\right.$ for ORI-PLA-RGD-NPs and $25.92 \pm 0.56 \mu \mathrm{g} \cdot \mathrm{mL}^{-1} \cdot \mathrm{h}$ for ORIPLA-NPs versus $11.89 \pm 0.35 \mu \mathrm{g} \cdot \mathrm{mL}^{-1} \mathrm{~h}$ for ORI solution), mean retention time (MRT) $(8.68 \pm 0.66$ hours for ORIPLA-RGD-NPs and $9.80 \pm 0.40$ hours for ORI-PLA-NPs versus $2.03 \pm 0.09$ hours for ORI solution), and apparent volume of distribution at steady state (Vss) $(14.91 \pm 1.14 \cdot \mathrm{L}$ $\mathrm{kg}^{-1}$ for ORI-PLA-RGD-NPs and $16.84 \pm 0.68 \mathrm{~L} \cdot \mathrm{kg}^{-1}$ for ORI-PLA-NPs versus $3.48 \pm 0.16 \cdot \mathrm{L} \mathrm{kg}^{-1}$ for ORI solution), and a decrease of clearance (CL) $\left(0.91 \pm 0.00 \mathrm{~mL} \cdot \mathrm{h}^{-1} \cdot \mathrm{kg}^{-1}\right.$ for ORI-PLA-RGD-NPs and $0.77 \pm 0.02 \mathrm{~mL} \cdot \mathrm{h}^{-1} \cdot \mathrm{kg}^{-1}$ for ORI-PLA-NPs versus $1.68 \pm 0.05 \mathrm{~mL} \cdot \mathrm{h}^{-1} \cdot \mathrm{kg}^{-1}$ for ORI solution). The differences in MRT, Vss, and CL between the ORI-PLA-RGD-NPs and the ORI-PLA-NPs were likely due to their distinct tissue distribution patterns, as shown in Figure 6. First, there was an apparent accumulation of ORI in tumor, liver, and spleen following the administration

Table I Pharmacokinetic parameters of oridonin (ORI) after a single injection of ORI solution, ORI-loaded atactic poly(D,L-lactic acid) nanoparticles (ORI-PLA-NPs), or ORI-PLA-NPs further modified by surface cross-linking with the peptide Arg-Gly-Asp (ORI-PLARGD-NPs) to mice bearing hepatocarcinoma 22 tumors

\begin{tabular}{llrc}
\hline Parameters & $\begin{array}{l}\text { ORI solution } \\
\text { group }\end{array}$ & $\begin{array}{l}\text { ORI-PLA-NP } \\
\text { group }\end{array}$ & $\begin{array}{l}\text { ORI-PLA-RGD- } \\
\text { NP group }\end{array}$ \\
\hline AUC $\left(\mu \mathrm{g} \cdot \mathrm{mL}^{-1} \cdot \mathrm{h}\right)$ & $1 \mathrm{I} .89 \pm 0.35$ & $25.92 \pm 0.56$ & $22.03 \pm 0.0 \mathrm{I}$ \\
$\mathrm{MRT}(\mathrm{h})$ & $2.03 \pm 0.09$ & $9.80 \pm 0.40$ & $8.68 \pm 0.66$ \\
$\mathrm{Vss}\left(\mathrm{L} \cdot \mathrm{kg}^{-1}\right)$ & $3.48 \pm 0.16$ & $16.84 \pm 0.68$ & $14.91 \pm 1.14$ \\
$\mathrm{CL}\left(\mathrm{mL} \cdot \mathrm{h}^{-1} \cdot \mathrm{kg}^{-1}\right)$ & $1.68 \pm 0.05$ & $0.77 \pm 0.02$ & $0.91 \pm 0.00$ \\
\hline
\end{tabular}

Abbreviations: $\mathrm{AUC}$, area under the concentration-time curve; $\mathrm{CL}$, clearance; h, hours; Vss, apparent volume of distribution at steady state; MRT, mean residence time.

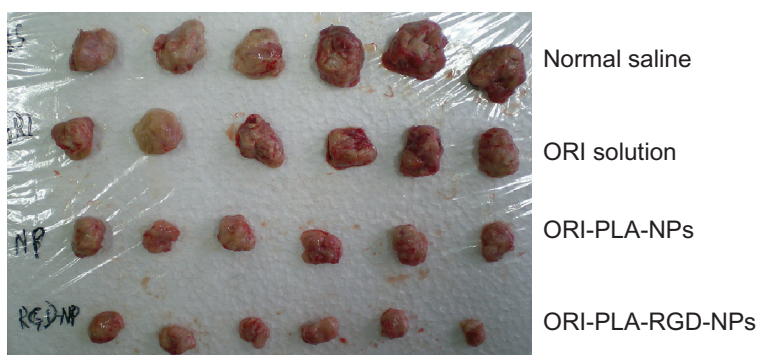

Figure 7 Tumors taken from mice treated for 10 days with normal saline, oridonin (ORI) solution, ORI-loaded atactic poly(D,L-lactic acid) nanoparticles (ORI-PLANPs), or ORI-PLA-NPs further modified by surface cross-linking with the peptide Arg-Gly-Asp (ORI-PLA-RGD-NPs).

of the NPs, which could be ascribed to the passive targeting effect. ${ }^{31}$ Second, the ORI-PLA-RGD-NPs showed a higher tumor-targeting efficiency than ORI-PLA-NPs, as reflected in the increased distribution of ORI in the tumor and corresponding decreased accumulation in the liver and tumor. This might be attributed to the improved active tissue targeting effect after RGD surface modification of the NPs. ${ }^{32}$

\section{Tumor inhibition effects in vivo}

To test the antitumor activity of the NPs, mice bearing $\mathrm{H} 22$ tumors were administered ORI in solution or as NPs for 10 days. The tumors removed from these animals are shown in Figure 7, and their mean weights and volumes are provided in Table 2. As seen in Figure 7, there is a manifest reduction of tumor volume in the groups treated with ORI-loaded NPs and a slight decrease in tumor volume in animals treated with ORI solution, compared with the normal saline group. The appearance of the tumors was in agreement with the statistical analysis of the tumor volume data, which showed that, when compared with the control group, all treatments significantly $(P<0.05)$ inhibited the tumor volume, with the biggest effect seen in the ORI-PLA-RGD-NP treatment group.

Table 2 In vivo antitumor effects of oridonin (ORI) solution, ORI-loaded atactic poly(D,L-lactic acid) nanoparticles (ORI-PLANPs), and ORI-PLA-NPs further modified by surface cross-linking with the peptide Arg-Gly-Asp (ORI-PLA-RGD-NPs)

\begin{tabular}{lll}
\hline Group & Tumor volume $\left(\mathbf{c m}^{3}\right)$ & Tumor weight $\mathbf{( g )}$ \\
\hline Normal saline & $1.05 \pm 0.32$ & $4.50 \pm 0.84$ \\
ORI solution & $0.71 \pm 0.27^{*, \mathrm{c}}$ & $3.27 \pm 0.44^{* *, \mathrm{~b}, \mathrm{c}}$ \\
ORI-PLA-NP & $0.50 \pm 0.1 \mathrm{I}^{* *}$ & $\mathrm{I} .95 \pm 0.40^{* *, \mathrm{a}, \mathrm{c}}$ \\
ORI-PLA-RGD-NP & $0.24 \pm 0.04^{* *, \mathrm{a}}$ & $\mathrm{I} .0 \mathrm{I} \pm 0.23^{* *, \mathrm{a}, \mathrm{b}}$ \\
\hline
\end{tabular}

Notes: Mean tumor volumes and weights are from groups of six mice injected once daily for 10 days. Mice were sacrificed 24 hours after the final dose. $* P<0.05$ and $* * P<0.0$ I compared with normal saline group; ${ }^{a}<0.01$ compared with ORI solution group; ' $P<0.01$ compared with ORI-PLA-NP group; ${ }^{c} P<0.01$ compared with ORI-PLA-RGD-NP group. 


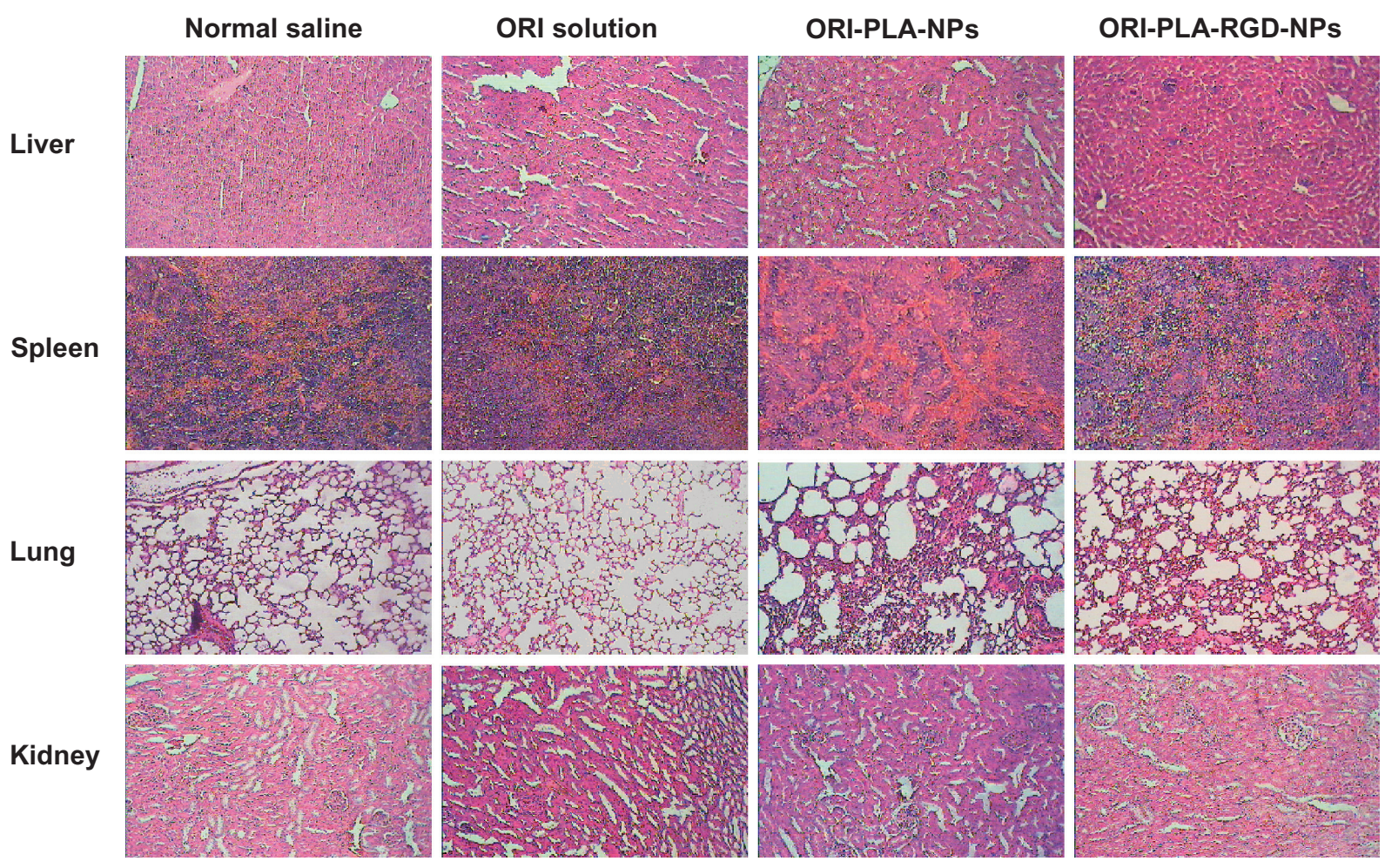

Figure 8 Representative images of hematoxylin and eosin-stained tissue sections from tumor-bearing mice treated with normal saline, oridonin (ORI) solution, ORI-loaded atactic poly(D,L-lactic acid) nanoparticles (ORI-PLA-NPs), or ORI-PLA-NPs further modified by surface cross-linking with the peptide Arg-Gly-Asp (ORI-PLA-RGD-NPs) (magnification 40x).

Similarly, the tumor weights were significantly $(P<0.01)$ inhibited by all three ORI treatments and, again, the effect was most pronounced in the ORI-PLA-RGD-NP treatment group. Interestingly, the data suggest the tumor weight was more sensitive to the treatment than the tumor volume. Representative pathological sections of liver, spleen, lung, and kidney after administration of normal saline, ORI solution, or ORI-loaded NPs are shown in Figure 8. There were no obvious pathological changes in the tissues of the treatment groups.

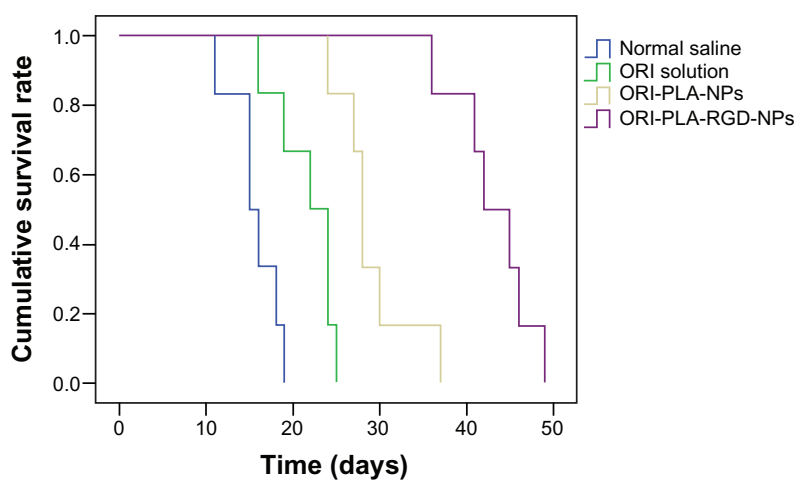

Figure 9 Survival curves of tumor-bearing mice treated once daily with normal saline, oridonin (ORI) solution, ORI-loaded atactic poly(D,L-lactic acid) nanoparticles (ORI-PLA-NPs), or ORI-PLA-NPs further modified by surface cross-linking with the peptide Arg-Gly-Asp (ORI-PLA-RGD-NPs).
The survival curves and median survival times obtained in the survival study are shown in Figure 9 and Table 3, respectively. As illustrated in Figure 9, the life spans of the H22 tumor-bearing mice were clearly prolonged by all three treatments, particularly by the ORI-PLA-RGD-NPs. This result was supported by the statistical analysis of the median survival times, which showed highly significant differences $(P<0.01)$ in the median survival times between the treatment groups and the normal saline group, as well as within the treatment groups.

Table 3 Survival times of tumor-bearing mice treated with oridonin (ORI) solution, ORI-loaded atactic poly(D,L-lactic acid) nanoparticles (ORI-PLA-NPs), or ORI-PLA-NPs further modified by surface cross-linking with the peptide Arg-Gly-Asp (ORI-PLARGD-NPs)

\begin{tabular}{ll}
\hline Group & Median survival time (days) \\
\hline Normal saline & $14.0 \pm 2.0$ \\
ORI solution & $21.0 \pm 2.0^{* *, \mathrm{~b}, \mathrm{c}}$ \\
ORI-PLA-NP & $27.0 \pm 0.6^{* *, \mathrm{a}, \mathrm{c}}$ \\
ORI-PLA-RGD-NP & $41.0 \pm 2.4^{* *, \mathrm{a}, \mathrm{b}}$ \\
\hline
\end{tabular}

Notes: Median survival times were determined for groups of twelve mice injected once daily with the indicated treatments. $* * P<0.01$ compared with normal saline group; ${ }^{a} P<0.01$ compared with ORI solution group; ${ }^{b}<<0.01$ compared with ORIPLA-NP group; ${ }^{c P}<0.01$ compared with ORI-PLA-RGD-NP group. 


\section{Conclusion}

In this study, ORI-loaded PLA-NPs were prepared with and without surface modification by RGD. The NPs were characterized physically and evaluated for ORI release in vitro. In vivo, the pharmacokinetics, tissue distribution, and antitumor activity of NPs were examined in $\mathrm{H} 22$ tumor-bearing mice. The results suggest that, compared with ORI-PLA-NPs and ORI solution, the tumor-targeting efficiency and subsequent antitumor efficacy of ORI may be improved by administration in the form of ORI-PLARGD-NPs.

\section{Acknowledgments}

This work was financially supported by project J50302 from the Shanghai Education Committee; projects 10ZR1430100, 11ZR1436500, and 10XD14303900 from the Science and Technology Commission of Shanghai Municipality, and projects NCET08-0898 and IRT1071 from the State Education Ministry, People's Republic of China.

The authors would like to thank Professor Sheng-Rong Guo from Shanghai Jiao Tong University, China, for his advice and assistance throughout the process of this study.

\section{Disclosure}

The authors report no conflicts of interest in this work.

\section{References}

1. Guan YZ, Wei TH. Clinical research on the oridonin injection for the interventional therapy of liver cancer. J Med Radiol Technol. 2005: 236-243. Chinese.

2. Ren KK, Wang HZ, Xie LP, et al. The effects of oridonin on cell growth, cell cycle, cell migration and differentiation in melanoma cells. J Ethnopharmacol. 2006;103(2):176-180.

3. Chen S, Gao J, Halicka HD, Huang X, Traganos F, Darzynkiewicz Z. The cytostatic and cytotoxic effects of oridonin (Rubescenin), a diterpenoid from Rabdosia rubescens, on tumor cells of different lineage. Int J Oncol. 2005;26(3):579-588.

4. Hsieh TC, Wijeratne EK, Liang JY, Gunatilaka AL, Wu JM. Differential control of growth, cell cycle progression, and expression of NF-kappaB in human breast cancer cells MCF-7, MCF-10A, and MDA-MB-231 by ponicidin and oridonin, diterpenoids from the Chinese herb Rabdosia rubescens. Biochem Biophys Res Commun. 2005;337(1):224-231.

5. Liu YQ, Mu ZQ, You S, Tashiro S, Onodera S, Ikejima T. Fas/FasL signaling allows extracellular-signal regulated kinase to regulate cytochrome c release in oridonin-induced apoptotic U937 cells. Biol Pharm Bull. 2006;29(9):1873-1879.

6. Zhang CL, Wu LJ, Zuo HJ, Tashiro S, Onodera S, Ikejima T. Cytochrome c release from oridonin-treated apoptotic A375-S2 cells is dependent on p53 and extracellular signal-regulated kinase activation. $J$ Pharmacol Sci. 2004;96(2):155-163.

7. Li D, Cui Q, Chen SG, et al. Inactivation of ras and changes of mitochondrial membrane potential contribute to oridonin-induced autophagy in a 431 cells. J Pharmacol Sci. 2007;105(1):22-33.

8. Wang H, Ye Y, Pan SY, et al. Proteomic identification of proteins involved in the anticancer activities of oridonin in HepG2 cells. Phytomedicine. 2011;18(2-3):163-169.
9. Cheng Y, Qiu F, Ye YC, Tashiro S, Onodera S, Ikejima T. Oridonin induces G2/M arrest and apoptosis via activating ERK-p53 apoptotic pathway and inhibiting PTK-Ras-Raf-JNK survival pathway in murine fibrosarcoma L929 cells. Arch Biochem Biophys. 2009;490(1): $70-75$.

10. Lou H, Gao L, Wei X, et al. Oridonin nanosuspension enhances antitumor efficacy in SMMC-7721 cells and H22 tumor bearing mice. Colloids Surf B Biointerfaces. 2011;87(2):319-325.

11. Hu HZ, Yang YB, Xu XD, et al. Oridonin induces apoptosis via PI3K/ Akt pathway in cervical carcinoma HeLa cell line. Acta Pharmacol Sin. 2007;28(11):1819-1826.

12. Cui Q, Tashiro S, Onodera S, Minami M, Ikejima T. Oridonin induced autophagy in human cervical carcinoma HeLa cells through Ras, JNK, and P38 regulation. $J$ Pharmacol Sci. 2007;105(4):317-325.

13. Xu W, Sun J, Zhang TT, et al. Pharmacokinetic behaviors and oral bioavailability of oridonin in rat plasma. Acta Pharmacol Sin. 2006;27(12): 1642-1646.

14. Zhang P, Liu Y, Feng N, Xu J. Preparation and evaluation of selfmicroemulsifying drug delivery system of oridonin. Int J Pharm. 2008; 355(1-2):269-276.

15. Liu Y, Zhang P, Feng N, Zhang X, Wu S, Zhao J. Optimization and in situ intestinal absorption of self-microemulsifying drug delivery system of oridonin. Int J Pharm. 2009;365(1-2):136-142.

16. Gao L, Zhang D, Chen M, et al. Studies on pharmacokinetics and tissue distribution of oridonin nanosuspensions. Int J Pharm. 2008;355(1-2): 321-327.

17. Zhang DR, Ren TC, Lou HX, Xing J. The tissue distribution in mice and pharmacokinetics in rabbits of oridonin-solid lipid nanoparticles. Yaо Хие Хие Bao. 2005;40(6):573-576. Chinese.

18. Xing J, Zhang D, Tan T. Studies on the oridonin-loaded poly(D,L-lactic acid) nanoparticles in vitro and in vivo. Int J Biol Macromol. 2007;40(2): 153-158.

19. Feng N, Wu P, Li Q, et al. Oridonin-loaded poly(epsilon-caprolactone)poly(ethylene oxide)-poly(epsilon-caprolactone) copolymer nanoparticles: preparation, characterization, and antitumor activity on mice with transplanted hepatoma. J Drug Target. 2008;16(6): 479-485.

20. Zhang X, McAuley KB, Goosen MFA. Towards prediction of release profiles of antibiotics from coated poly(DL-lactide) cylinders. J Control Release. 1995;34(2):175-179.

21. Yolles S, Leafe TD, Woodland JH, Meyer FJ. Long acting delivery systems for narcotic antagonists II: release rates of naltrexone from poly(lactic acid) composites. J Pharm Sci. 1975;64(2): 348-349.

22. Xiao RZ, Zeng ZW, Zhou GL, Wang JJ, Li FZ, Wang AM. Recent advances in PEG-PLA block copolymer nanoparticles. Int J Nanomedicine. 2010;5:1057-1065.

23. Hu Z, Luo F, Pan Y, et al. Arg-Gly-Asp (RGD) peptide conjugated poly(lactic acid)-poly(ethylene oxide) micelle for targeted drug delivery. J Biomed Mater Res A. 2008;85(3):797-807.

24. Cameron DJ, Shaver MP. Aliphatic polyester polymer stars: synthesis, properties and applications in biomedicine and nanotechnology. Chem Soc Rev. 2011;40(3):1761-1776.

25. Yuan W, Yuan J, Zheng S, Hong X. Synthesis, characterization, and controllable drug release of dendritic star-block copolymer by ringopening polymerization and atom transfer radical polymerization. Polymer. 2007;48(9):2585-2594.

26. Murakami H, Kobayashi M, Takeuchi H, Kawashima Y. Preparation of poly(DL-lactide-co-glycolide) nanoparticles by modified spontaneous emulsification solvent diffusion method. Int J Pharm. 1999; 187(2):143-152.

27. Yoo HS, Oh JE, Lee KH, Park TG. Biodegradable nanoparticles containing doxorubicin-PLGA conjugate for sustained release. Pharm Res. 1999;16(7):1114-1118.

28. Yoo HS, Lee KH, Oh JE, Park TG. In vitro and in vivo anti-tumor activities of nanoparticles based on doxorubicin-PLGA conjugates. J Control Release. 2000;68(3):419-431. 
29. Saxena V, Sadoqi M, Shao J. Indocyanine green-loaded biodegradable nanoparticles: preparation, physicochemical characterization and in vitro release. Int J Pharm. 2004;278(2):293-301.

30. Hu LD, Tang X, Cui FD. Preparation of solid lipid nanoparticles loaded with all-trans retinoic acid and their evaluation in vitro and in vivo. Yao Xие Xие Baо. 2005;40(1):71-75. Chinese.
31. Dinarvand R, Sepehri N, Manoochehri S, Riuhani H, Atyabi F. Polylactide-co-glycolide nanoparticles for controlled delivery of anticancer agents. Int J Nanomedicine. 2011;6:877-895.

32. Hersel U, Dahmen C, Kessler H. RGD modified polymers: biomaterials for stimulated cell adhesion and beyond. Biomaterials. $2003 ; 24(24): 4385-4415$.

\section{Publish your work in this journal}

The International Journal of Nanomedicine is an international, peerreviewed journal focusing on the application of nanotechnology in diagnostics, therapeutics, and drug delivery systems throughout the biomedical field. This journal is indexed on PubMed Central, MedLine, CAS, SciSearch $®$, Current Contents $® /$ Clinical Medicine,
Journal Citation Reports/Science Edition, EMBase, Scopus and the Elsevier Bibliographic databases. The manuscript management system is completely online and includes a very quick and fair peer-review system, which is all easy to use. Visit http://www.dovepress.com/ testimonials.php to read real quotes from published authors.

Submit your manuscript here: http://www.dovepress.com/international-journal-of-nanomedicine-journal 